Picture Bandwidth Compression

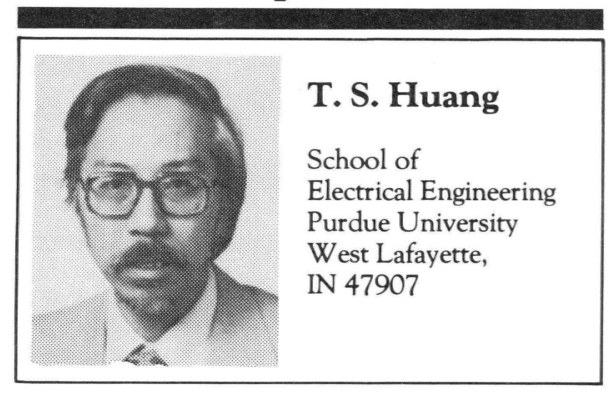

\section{Efficient Image Coding}

In many applications, one needs to transmit or store images in digital form. The number of bits involved is of ten astronomical. It is desirable and in many cases mandatory to compress or efficiently code the data.

In the past, much of the research in image coding was motivated by video phone. More recently, attention has been shifted to teleconference and studio television applications. Straight digitization of an image requires about 8 bits per picture element (pel). Using in-frame coding techniques (DPCM, transform coding), one can reduce the bit rate to around 1 to 2 bits per pel which preserves good picture quality. Further reduction is possible if one uses frame-to-frame coding techniques where the correlation between frames is utilized. A way of doing that is to transmit frame differences. Obviously, if there is no motion, the differences will be very small (zero, if there is no noise). But if there is a lot of motion, the differences can be large. An area of active current research is motion-compensated coding. The motion is detected and estimated. Then motion-compensated frame differences are transmitted. A word of caution is in order here. Most coding schemes degrade the image quality. Therefore, the bit rate or compression factor of a coding method is meaningless by itself. One has also to look at the received images to see how much degradation has been incurred.

Another problem where efficient coding is badly needed is the storage and transmission of multispectral LANDSAT images. It is estimated that each of LANDSATS 1,2 , and 3 creates approximately $1.5 \times 10^{13}$ bits/year. LANDSAT D with its higher resolution images will create approximately $3.7 \times 10^{15}$ bits/year. A large compression factor is needed to facilitate the data handling. Since different users of these multispectral images are to ex tract different types of information from them, it is difficult to decide what degradations of the data are acceptable. To be on the safe side, one will probably demand essentially no degradation from the original digitized data. This makes large compression impossible.

A completely different class of images is graphics, which includes business letters and documents, engineering drawings, wea ther maps, etc. These are images of man-made symbols. Most of these images are nominally two-level, i.e., each pel is either black or white-there is no need for intermediate gray levels. The digital storage and transmission of these images have become increasingly important, because of the intensive current interest in office automation. Most coding methods of digital graphics are based on runlength coding and its extensions. However, the current trend in in exploring the use of pattern recognition techniques.

Perhaps the best way to find out the state of the art and current research and development activities in efficient image coding is to attend the international Picture Coding Symposium held approximately every 1 or 2 years. A report on the last meeting (July 1979) by Professors Sabri and Dubois of Canada follows.

1979 Picture Coding Symposium (PCS)

M. S. Sabri, Bell-Northern Research

E. Dubois, INRS Telecommunications

The Picture Coding Symposium is an international meeting for picture coding professionals from industries, universities, and government research institutions. It is held approximately every two years. Researchers are encouraged to report on new ideas as well as on research and development work currently in progress.

The 1979 Picture Coding Symposium was held in Ipswich, England, July 9-1 1, 1979. One hundred and twenty-three participants from Canada, U.S.A., Europe, and Japan attended. There were three full days of sessions on various aspects of picture coding. The main topics covered were: human observer, coding of graphics, intraframe and interframe coding, picture processing and effects of transmission errors.

The next Picture Coding Symposium will be held in Montreal in June 1981

A summary of the main themes, trends, and pertinent papers follows.

\section{Human Observer}

Major products include polarizing prisms and cubes, infrared optics, cylinder lenses, and a full line of mechanical devices.
Write for Catalog
Glan-Lasers provide efficient polarization at higher energy levels, with minimum reflection losses at the air-spaced surfaces. Series of design types range from low energy density versions, thru medium energy single escape, to high density $Q$ switch double escape and special Brewster Angle prisms.

Other polarizing prisms include Glan Thompson, Glan Taylor, Ahrens, Rochon, Wollaston, and Beam Splitters.

Call or write - we're here to serve you.

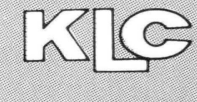

KARL LAMBRECHT CORPORATION 4204 North Lincoln Ave. • Chicago, III. 60618 U.S.A. Phone: (312) 472-5442 TELEX: 254771 Lambrecht Cgo.
Several papers that deal $t$ with the masking effects in television signals were presented. Measurements of 2-D edge masking functions on monochrome, television were reported. In addition two aspects of temporal masking were presented, i.e., factors involved in the masking effects by a moving object on newly exposed background, and the masking of the chrominance transitions by the luminance signal.

\section{Slow Scan TV Picture Coding}

This session reflects the increasing interest in slow scan TV systems. It was the first time that the PCS devoted a full session to discuss the 
coding of picture material in such systems. The session dealt with coding of still pictures for transmission over narrowband communications links (1.2 to $9.6 \mathrm{kbits} / \mathrm{sec})$. Several techniques were presented, namely DPCM, orthogonal transforms, and bilevel coding. For the DPCM system a 3 bit quantizer is used, in addition to error detection and concealment at the receiver. The second approach utilizes orthogonal transformations. Three types of transforms were investigated, namely Hadamard, cosine, and Haar transforms. Lower order transform coefficients are transmitted first and the picture is allowed to build up gradually. For color PAL signals, component coding is utilized as composite coding was judged to be more complex to implement.

The third approach involves time sequential coding of dithered binary pictures. The continuous tone (multilevel) picture is converted to a bilevel picture using the ordered dither technique. Binary picture elements are transmitted in different order depending on the type of picture material. Further reduction in transmission time was proposed using runlength and predictive coding.

Although transform coding techniques seem to be the most obvious choice for coding of picture material in such systems, the complexity of the receiver terminal is still relatively high. DPCM systems offer simpler structures, however they have poor transmission channel error propagation characteristics. The bilevel coding using the ordered dither technique may not be suitable for display on TV monitors because of flicker problems.

\section{Interframe Coding}

Different topics on interframe coding of video signals were handled in several sessions. The two main topics that received attention were temporal filtering and movement compensated predictors. Both have immediate potential application on codecs for video-conferencing.

\subsection{Temporal Filtering}

Four papers were presented on this topic. They involved the utilization of temporal filtering techniques to reduce the bit rate, suppress noise, and reduce the effects of aliasing when temporal subsampling is used. An adaptive nonlinear temporal prefilter, which utilizes information from the current frame difference signal as well as the previous frame difference signal to perform the temporal filtering operation, was reported. In addition, two papers on the use of nonlinear temporal filtering for bit rate reduction and noise suppression were presented. Results of a study on different aspects of linear and nonlinear temporal filtering of the NTSC video signals were also reported.

\subsection{Movement Compensation}

Movement compensated coding is receiving great attention from many research groups concerned with in terframe coding. Several papers describing techniques for interframe coding using movement compensation were presented. A pel recursive algorithm, which operates on a pel or small block basis to estimate a moving object displacement from one frame to the next, was presented. A movie portraying the performance of this algorithm and contrasting it with conventional previous frame prediction was shown.

In another paper, displacement estimates were obtained to an integer number of pels by correlation techniques. A key point made during this paper was that temporal subsampling can be
X-ray image before processing

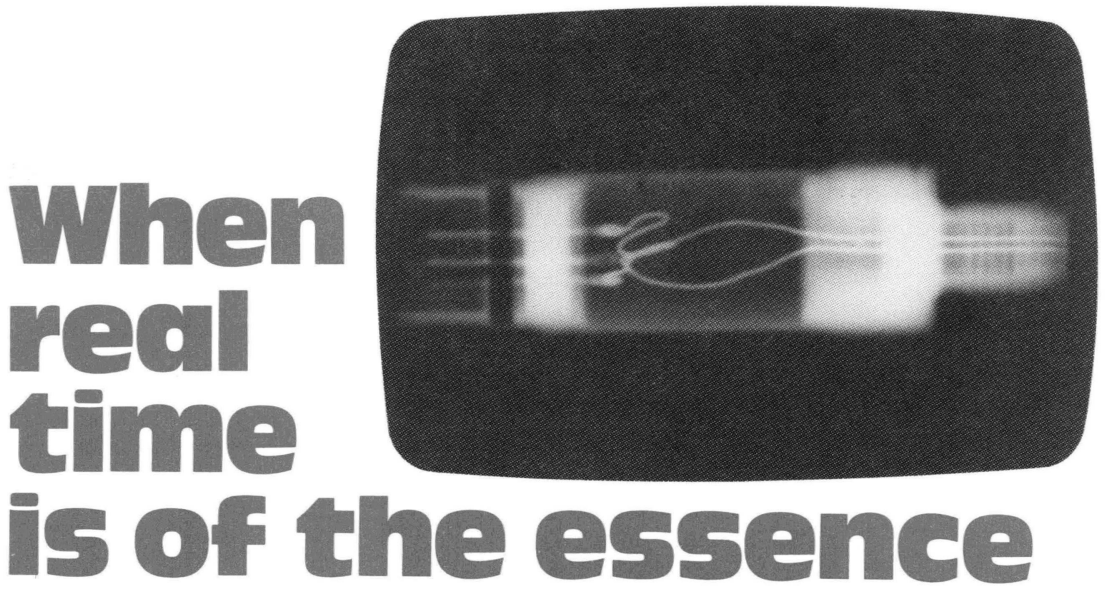

\section{Quentex Digital Video Processors}

In the real world of video processing, images move much faster than traditional A/D converter, computer and software video combinations. Quantex beats the time lag between the video event and pro-

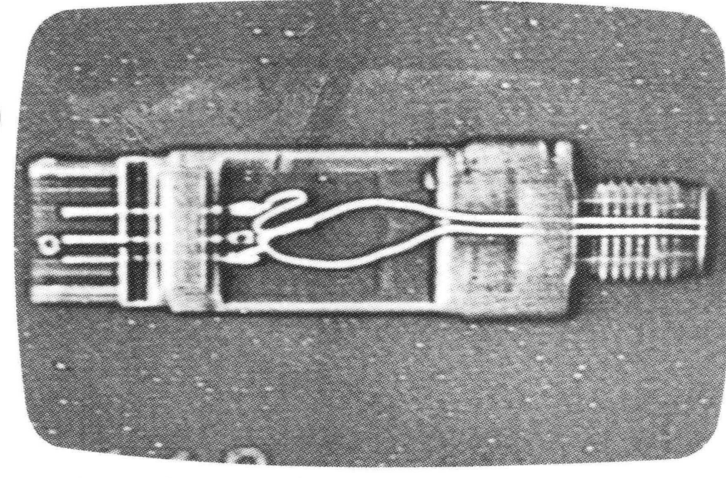
$X$-ray image after processing cessed image by simultaneously inputting, computing and outputting, up to 10 million pixels per second transferring an entire picture in 1/30th of a second.

At this real time rate, Quantex digital video processors can:

- reduce noise by summing or averaging frames; as with a time exposure with film,

- eliminate background or compare images to a standard by subtracting frames,

bring out subtle contrast variations by expanding part of the grey scale,

- enhance edges, and

store a transient image

Whether you need stand alone performance or pre-processing for quicker data transfer and computer

handling of image data, Quantex lets you instantly improve video quality, process a changing image, eliminate off-line delays, automate imaging, and reduce the use of film. Contact Quantex today for the whole picture.
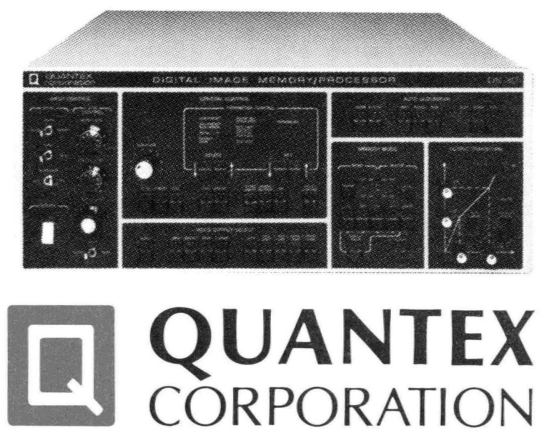

252 No. Wolfe Road

Sunnyvale, CA 94086 • (408) 733-6730 used with movement compensated prediction because of the reduction of the effective temporal bandwidth.

In addition, results of real-time coder simulators, utilizing movement compensated predictors were also presented.

\section{Coding of Graphics}

Several papers describing coding and processing methods of graphic signals were presented. Among them, a paper describing techniques for efficient coding of newspaper facsimile pictures. The image is segmented into text and screened photograph sections. The text is coded using conventional facsimile coding, while the photographic data is coded with an adaptive predictive coder, which estimates the periodicity of the screen. This technique gives up to double the compression ratio for pages which are largely photographic.

A dual mode OCR/PR facsimile system being developed was described. Compressions of 50 to 1 was reported. When operating in OCR mode only, i.e., rejecting all unrecognizable material, compressions of 250:1 can be obtained. Results of a subjective experiment of the effects of preprocessing techniques on digital facsimile signals was reported. 


\section{The History of Optics}

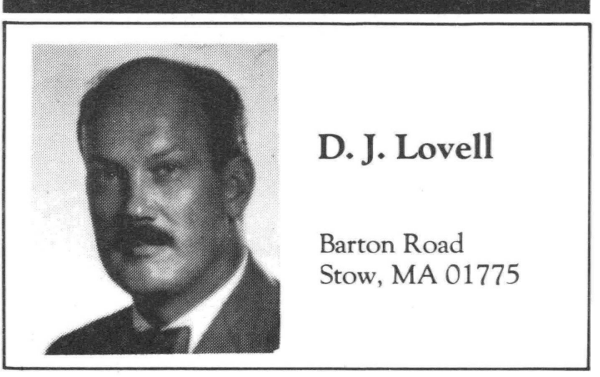

\section{The Evolution of Optical Technology}

Technology is sometimes considered to be the handmaiden of science. However one considers their association, it is a close, important, and necessary relationship. Early optical instruments were devised for utilitarian purposes, primarily as an aid to astronomical observations. These observations, in turn, permitted more accurate measurements of stellar positions to be made which led to an improved understanding of the passage of light through the atmosphere. From this, it was possible to unlock the mysteries of the rainbow, the colors of the clouds and sky, and other properties of the nature of light. All this took time, of course, and required a continual improvement in optical technology.

\section{The Gnomon}

Undoubtedly the oldest optical instrument was the simple gnomon, which consisted of a post erected upright in the ground. The direction and length of the shadow cast by this device varied according to the time of day and to the season of the year. Ptolemy used a gnomon to determine the times of equinoxes and solstices. Probably, the pyramids of Egypt served as a gigantic gnomon.

A portable gnomon was made using a hollow hemispheric bowl with a small vertical pointer at the center. Lines were engraved on the inner surface of the bowl to mark the position of the shadow at different hours of the day.

\section{The Merkhet}

Another simple instrument was the merkhet which consisted of a strip of wood into which a narrow, deep, V-shaped notch was cut at one end. A plumb line was used in conjunction with this to determine the culmination of a particular star. This was accomplished by placing the plumb line at some distance from the V-notched stick and noting the time when the star being observed was viewed exactly in line with the notch and plumb line. Such an instrument was used by the Egyptians some 5000 years ago.

\section{The Armillary}

A more sophisticated instrument serving approximately the same purpose as the merkhet was the meridional armillary. This device consisted of a bronze ring accurately made with a uniform cross section, and having a diameter of $25 \mathrm{~cm}$ or more. The ring was clamped onto a fixed pillar. The lateral face of the ring was graduated into $360^{\circ}$, and each degree was subdivided in to $5^{\prime}$. Note that if the ring were $50 \mathrm{~cm}$ in diameter, a $5^{\prime}$ division would be spaced by about $0.36 \mathrm{~mm}$. Inside the fixed ring a smaller ring was fitted so that it could be rotated in contact with the fixed outer ring. Small plates were fixed at opposite ends of a diameter of this inner ring to serve as sights, so that both the time of conjunction and the altitude could be measured.

Both the meridional armillary and the merkhet were necessarily aligned accurately to the plane of the meridian. This could be determined by measuring the shortest shadow of a gnomon. Although the merkhet could, in principle, be used to determine altitudes, it would be far easier to do so with the armillary. This was used to find the height of the winter and summer solstices.

\section{The Parallel Instrument}

Another device of interest was the parallactic instrument which was used to measure the zenithal distances of stars and the moon at the time of culmination. This, in effect, is the ancestor of the modern transit. It consisted of a vertical bar, over two meters in height. At the top of this was hinged a wooden spar sufficiently thick to be rigid and of about the same length as the bar. At the bottom of the bar was hinged by a sliding couple a boom which was also engaged to the free end of the spar. A star was observed by aligning the spar to it. The triangle formed by these three members could then be solved to give the zenithal distance.

Oddly enough, Ptolemy was not aware that this determination could be simplified by placing graduated marks on the boom. That was accomplished by an Arabian astronomer in the ninth century.

Optical instruments used for astronomical observations generally do not require that they be portable. Hence, a number of observatories existed in medieval times. These were hardly more than ordinary houses situated in an open position so as to command a good view of the horizon. They were, therefore, located away from metropolitan areas. Note that we still locate observatories in remote locations, but for a different reason.

\section{The Clepsydra}

Aside from its in terest to astrologists, astronomy was studied primarily for the purpose of constructing calendars and for determining terrestrial positions (latitude and longitude). Of course it was necessary to utilize clocks to aid these measurements. The clepsydra, or water clock, was probably the basic instrument for the determination of time at night during ancient times. It consisted of a container of water fed by a constant flow. A float rising with the water marked the passage of time. These instruments were probably accurate to within 10 minutes. The calendar of Omar Khayyam of 1079 was as accurate as any until the Gregorian reform in 1852 .

\section{The Astrolabe}

The determination of latitude was generally accomplished by the use of an astrolabe. This, of course, would be of great consequence for a traveler. Determining longitude, on the other hand, also requires a knowledge of time. This can readily be determined by a stationary observer, but presents some difficulties to a traveler. 
Although the astrolabe was known to Ptolemy, its widespread use dates to about the tenth century. One of the early descriptions of the astrolabe was written by Geoffrey Chaucer in the fourteenth century. His Tretis of the Astrolabie was addressed to Ltel Lowys my sone and states I nam but a lewd compilatour of the labour of olde Astrologiens.

The astrolabe is a device for measuring the angular distance between two objects. It consists of a graduated circular plate with a datum line and a rotatable pointer called the alidade on which are located two sights. The astrolabe is hung from a ring at the top of the diameter perpendicular to the datum line. The alidade is rotated until it points at a particular star, whose altitude can now be read.

However, the instrument does far more than merely determine altitudes. The celestial sphere is inscribed on the side of the astrolabe opposite to that supporting the alidade. This sphere is formed by projecting it from its south pole on to the plane of the equator. Thus, the solstital circles and the equator are mapped concen trically. The rim of the astrolabe is made to coincide with the winter solstital circle and is divided in to 360 degrees to permit measuring right ascension, i.e., equinoctial time.

Knowing the longitude of the sun (e.g., from a table) permits one to ascertain his position on earth. This feature of the astrolabe is useful only near the latitude for which it was prepared, but different plates could be substituted for various latitudes enabling a traveler to use the instrument over prolonged journeys.

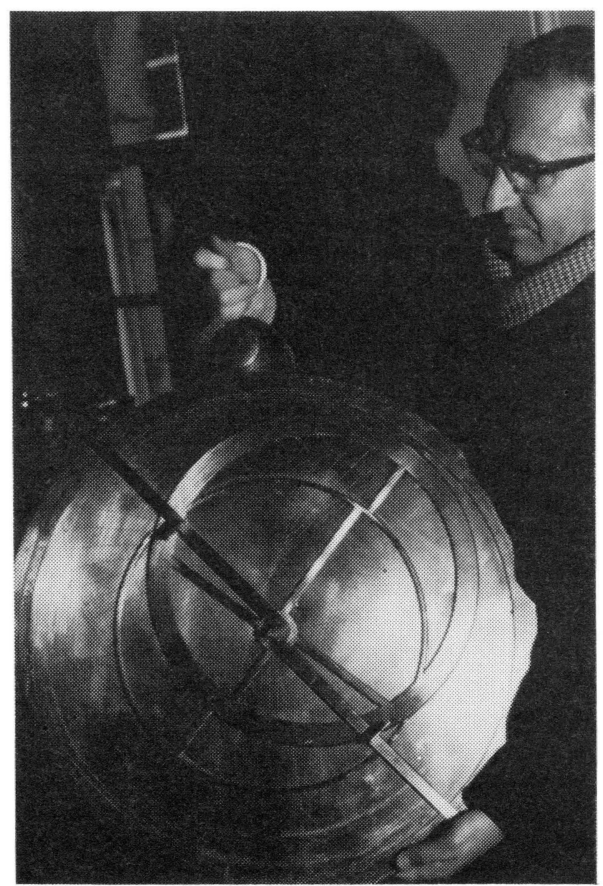

Astrolabe made in $\mathbf{1 5 7 5}$ by Humphrey Cole. Photographed at St. Andrews University, Scotland, by D. J. Lovell.

It is interesting to note that in addition to its use as a navigational tool and astronomical in- strument, the astrolabe could be used to cast horoscopes, to survey, to perform some calculations, and to undertake quite a variety of other tasks. Nevertheless, the determination of time was still an obstacle to its use over long journeys.

\section{The Necessaire}

Sun dials, of course, can be used to determine time, but these are not portable unless they can be combined with a means of locating north. The compass was not readily available until the end of the fifteenth century. By that time, the necessaire came into use. This was a compact device that could be used as an astrolabe, compass, sun dial, quadrant, calendar, calculator, and a moon phase reckoner. One can imagine the convenience this provided a traveler!

\section{The Ring Dial}

The ring dial is another example of optical instruments in use prior to the Renaissance. It is simply a small ring to which are attached sufficient surfaces to serve as a sun dial at any latitude, providing of course that one knew how to locate north. This could be ascertained by locating the North Star, establishing a meridian, and waiting for daybreak prior to its use. The ring dial receives its importance from its small size (perhaps $10 \mathrm{~cm}$ in diameter) and consequent portability.

\section{The Eye Glass}

Optical technology was also making its impact in other areas during the Middle Ages. By the end of the thirteenth century, spectacles were in use.

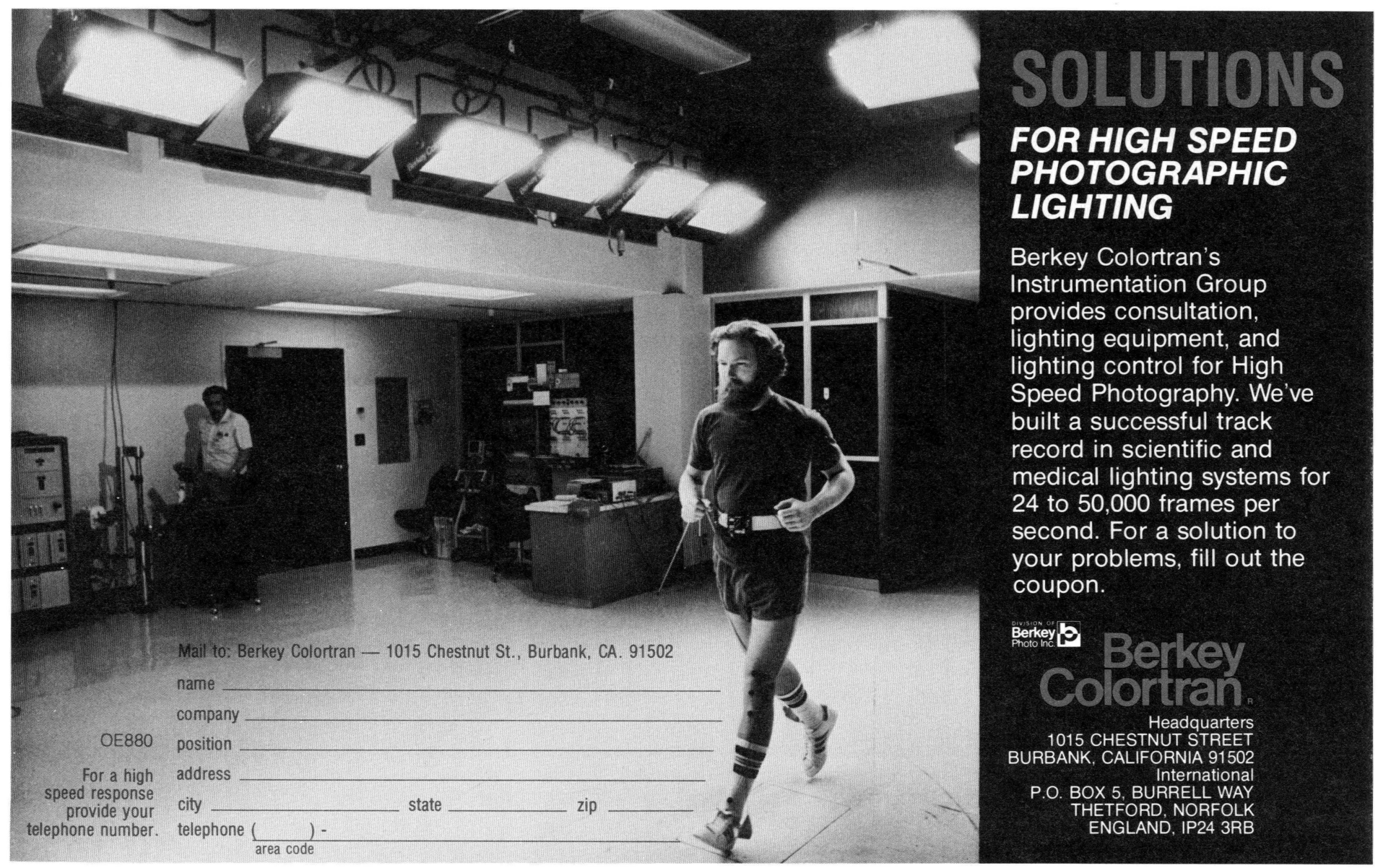


It is recorded that a certain Friar of Pisa preached a sermon in 1306 in which he claimed that he had spoken to the man who had invented, some 20 years previously, eye glasses which make for good vision. A portrait of the venerable Hugues de St. Cher wearing spectacles while seated at a desk copying a book was painted by Tommaso of Medina in 1352. This is believed to be the first portrait of a man wearing spectacles.

The inventor of spectacles is shrouded in mystery possibly because the inventor (if one existed) was supported by a duke or other noble and it would not be prudent to bring the discovery to the attention of others and thereby invite competition.

Initially, only convex lenses were used in spectacles. These correct presbyopia (the inability to fully accomodate from which many of us suffer with age). Reference to the use of concave lenses in spectacles to correct myopia (short sightedness) was not made prior to the midfifteenth century. In any event, the discovery of the utility of spectacles does not seem to have been accompanied by significant investigations of either the theory of lenses or of the functioning of vision.

The optical devices described here were precursors of those that would provide data enabling our knowledge of the nature of light and of the universe as well to be impressively enlarged. Until the mid-six teenth cen tury these devices aided man on his travels, his knowledge of the earth and solar system, and his ability to improve his vision. But it did so with limited precision. At that time, technology had improved sufficiently to enable optical science to make remarkable advances.

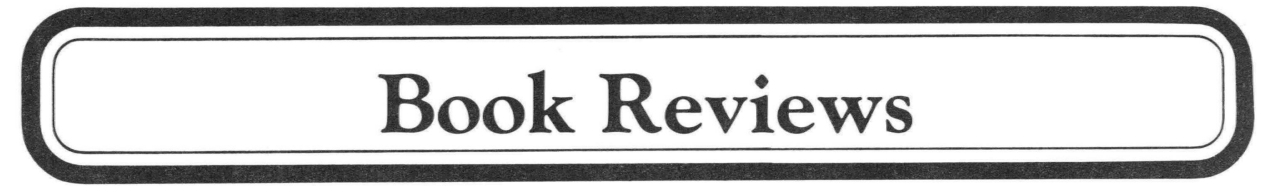

REMOTE SENSING OF EARTH FROM SPACE: ROLE OF "SMART SENSORS," Vol. 67 Progress Series, Roger A. Breckenridge, Ed. 503 pp., illustrated, bibliography, index. ISBN 0-915928-33-7. American Institute of Aeronautics and Astronautics, 1290 Avenue of the Americas, New York NY 10019 (1979) \$42.50.

Reviewed by H. John Caulfield, Aerodyne Research, Inc., Bedford Research Park, Bedford, MA 01730 .

It is not your editor's policy to review books himself. In this case I made an exception for two reasons. First, the subject matter is of great interest to me. Second, the reviewer originally assigned this book returned it to me (with apologies) after several months. In retrospect I am glad to have this opportunity to add this useful book to my personal library.

This book differs from the myriad of others on remote sensing in its emphasis on the architecture of "smart" sensors. Smart sensors incorporate on-board processing for such tasks as pattern recognition, adaptation to changing conditions, and downlink bandwidth compression. Most of the chapters in this book were papers at an AIAA/NASA conference on smart sensors at NASA Langley Research Center in late 1978.

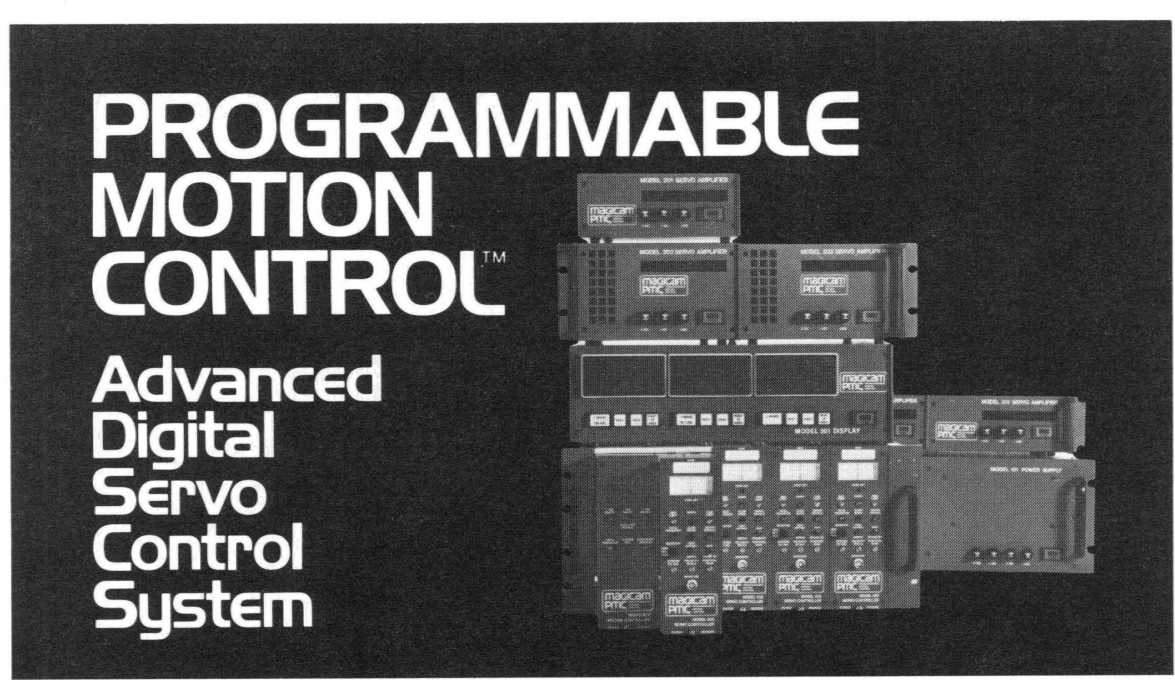

\section{Where Performance and Accuracy Are Really Vital}

A REMARKABLE SYSTEM, FEATURING: Operation in a sampled data environment. High dynamic accuracy. Feed forward error modeling. Digital vector desampling. Modular expandability. Dynamically variable sample rates.

Thoroughly proven and already operational in the Aerospace, Motion Picture and Television industries.

Write for our system brochure:

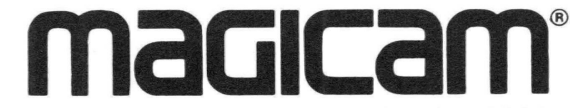

5451 Marathon Hollywood, California 90038
This volume is divided into five chapters. All but the first chapter are comprised of multiple papers relevant to the chapter contents. The chapters are:

I. Introduction

II. User Concepts

III. Data Processing Systems

IV. Advanced Device Technology

V. Data Preprocessing Techniques

The introduction by the editor, Roger A. Breckenridge, and an associate, Charles Husson, gives an implicit definition of the fuzzy field of smart sensors and shows the technical and economic reasons that "the authors see the use of smart sensors gradually increasing through 1990 and becoming quite significant around the year 2000.'

Chapter II, User Concepts, has contributions on the potential uses of smart sensors by the Interior Department, the Coast Guard, and in agriculture. It proceeds the description of specific sensors for earth resources, ocean observation, landmark identification, etc.

Chapter III, Data Processing Systems, has five papers of the NASA End to End Data System (NEEDS). "End-to-end" apparently connotes an integrated, massively parallel attack on the problem loosely diagrammed below.

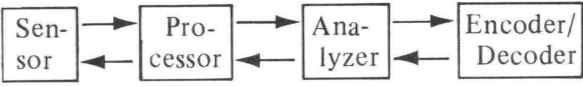

A favorite paper of mine in that chapter was a JPL report by Wellman and Norris on "Onboard Processing for Future Space-Borne Imaging Systems" which contained enough detail to allow the reader real insight into the design. Another JPL paper, "Smart Sensors for Smart Hands" by Bejczy had no bearing on "Remote Sensing from Space" but describes some marvelous sensors.

Chapter IV, Advanced Device Technology, concentrates on CCD arrays but it also includes SAW, holography, DEFT, in tegrated optics, and GaAs integrated circuits. While none of this material appears to be unavailable elsewhere, their inclusion here is very useful.

Chapter V, Data Preprocessing Techniques, is an excellent collection of systems and "tricks" especially related to detector arrays.

This book will be useful to all workers in smart sensors. If these authors are correct in their predictions, that will eventually include a great many of us.

Correction: The reviewer of $A$ Handbook of Optical Holography [Optical Engineering 19(3), SR-089 (May/June 1980)] was Elliot Eichen, graduate student, Optical Sciences Center, University of Arizona, not James C. Wyant as stated. 